PROCEEDINGS OF THE

AMERICAN MATHEMATICAL SOCIETY

Volume 128, Number 4, Pages 1191-1196

S 0002-9939(99)05609-9

Article electronically published on December 10, 1999

\title{
LARGE VOLUME GROWTH AND FINITE TOPOLOGICAL TYPE
}

\author{
D. ORDWAY, B. STEPHENS, AND D. G. YANG \\ (Communicated by Christopher Croke)
}

\begin{abstract}
It is shown in this paper that a complete noncompact $n$-dimensional Riemannian manifold with nonnegative Ricci curvature, sectional curvature bounded from below, and large volume growth is of finite topological type provided that the volume growth rate of the complement of the cone of rays from a fixed base point is less than $2-1 / n$.
\end{abstract}

\section{INTRODUCTION}

A complete Riemannian manifold with nonnegative sectional curvature is necessarily of finite topological type. This follows from the celebrated Cheeger-Gromoll soul theorem [4]. However, complete Riemannian manifolds with nonnegative or positive Ricci curvature and bounded sectional curvature may have infinitely generated homology group as demonstrated by the examples of J.P. Sha and the third author in $[8$. Thus the topological type of these manifolds may be infinite. This leads to the interesting problem: Under what additional conditions are the manifolds of finite topological type? The first result in this direction is due to Abresch and Gromoll in [1] where they assumed an additional condition on the diameter growth of the manifolds. Z.M. Shen and G.F. Wei [7] also have some results in this direction. Recently, Z.M. Shen has shown an interesting finite topological type theorem in 6] for manifolds with large volume growth and the complement of the cone of rays from a fixed base point has linear volume growth.

The aim of this note is to prove the following finite topological type result.

Theorem 1. Let $(M, g)$ be a complete noncompact $n$-dimensional Riemannian manifold with nonnegative Ricci curvature and large volume growth. Assume that

$$
\operatorname{Vol}(B(p, r))=\alpha r^{n}+o\left(r^{2-1 / n}\right)
$$

where $p \in M$ is any fixed base point and $\alpha$ is a positive constant. If, in addition, the conjugate radius of $M$ is bounded from below by a positive constant or the sectional curvature is bounded from below, then $M$ has finite topological type.

After the completion of this paper, we received a preprint from Dr. X. Menguy [5] where he has shown by examples that, for each $n \geq 4$, there are $n$-dimensional

Received by the editors January 11, 1998.

1991 Mathematics Subject Classification. Primary 53C21.

Key words and phrases. Excess function, finite topological type, large volume growth, nonnegative Ricci curvature, Riemannian manifold, volume comparison theorem.

This research was partially supported by NSF grant DMS97-32058. 
manifolds with infinite topological type that admit complete Riemannian metrics with nonnegative Ricci curvature, bounded sectional curvature, and large volume growth. Thus the additional growth condition on the lower order term $o\left(r^{2-1 / n}\right)$ in Theorem 1 cannot be dropped for $M$ to be of finite topological type. However, it is not clear whether the growth rate $2-1 / n$ is the best possible.

Finally, we would like to thank Dr. X. Menguy for sending us his preprint and thank the referee for his suggestions.

\section{Preliminaries}

Throughout this paper, $M$ will be a complete noncompact $n$-dimensional Riemannian manifold with nonnegative Ricci curvature. Fix a base point $p \in M$. Let $B(p, r) \subset M$ be the closed ball of radius $r$ centered at $p$ and let $\operatorname{Vol}(B(p, r))$ be its volume.

Lemma 2. The limit

$$
\alpha_{M}=\lim _{r \rightarrow \infty} r^{-n} \operatorname{Vol}(B(p, r)) \geq 0
$$

exists and it is independent of the base point $p \in M$. Moreover, for each point $q \in M$ and all $r \geq 0$, we have

$$
\operatorname{Vol}(B(q, r)) \geq \alpha_{M} r^{n}
$$

Proof. By the Bishop-Gromov volume comparison theorem [2], the function $r^{-n} \operatorname{Vol}(B(p, r))$ is a nonincreasing nonnegative function. Thus the limit exists. To show that the limit is independent of the base point $p$, notice that, by the triangle inequality, we have

$$
B(p, r) \subset B(q, r+d)
$$

for any point $q \in M$, where $d=d(p, q)$ is the distance from $p$ to $q$. It follows that

$$
\begin{aligned}
\lim _{r \rightarrow \infty} r^{-n} \operatorname{Vol}(B(q, r)) & =\lim _{r \rightarrow \infty}(r+d)^{-n} \operatorname{Vol}(B(q, r+d)) \\
& \geq \lim _{r \rightarrow \infty} r^{n}(r+d)^{-n} r^{-n} \operatorname{Vol}(B(p, r)) \\
& =\lim _{r \rightarrow \infty} r^{-n} \operatorname{Vol}(B(p, r)) .
\end{aligned}
$$

Now permuting $p$ with $q$ yields the opposite inequality. Hence

$$
\lim _{r \rightarrow \infty} r^{-n} \operatorname{Vol}(B(q, r))=\lim _{r \rightarrow \infty} r^{-n} \operatorname{Vol}(B(p, r)) .
$$

The inequality now follows from the fact that $\alpha_{M}$ is independent of the center of the ball and $r^{-n} \operatorname{Vol}(B(q, r))$ is a nonincreasing function.

Definition 3. $M$ is said to have large volume growth if $\alpha_{M}>0$.

Let $S_{p} M \subset T_{p} M$ be the unit sphere in the tangent space $T_{p} M$ of $M$ at $p$. For any subset $N \subset S_{p} M$, let

$C(N)=\left\{q \in M \mid\right.$ there is a minimal geodesic $\gamma$ from $p$ to $q$ such that $\left.\gamma^{\prime}(0) \in N\right\}$

be the cone over $N$ and let

$$
B(N, r)=B(p, r) \cap C(N) .
$$

Notice that, by definition, $C(N)$ and $C\left(N^{c}\right)$ are disjoint subsets of $M$ for any $N \subset S_{p} M$. The same is true for $B(N, r)$ and $B\left(N^{c}, r\right)$. Let

$$
\Sigma=\left\{v \in S_{p} M \mid \exp _{p}(r v):[0, \infty) \rightarrow M \text { is a ray }\right\} .
$$


It is easy to see that $\Sigma$ is a closed subset of $S_{p} M$, thus $\Sigma^{c}=S_{p} M \backslash \Sigma$ is an open subset of $S_{p} M$. With these notations, it is clear that $C(\Sigma)$ is the cone of rays from $p$ and $C\left(\Sigma^{c}\right)$ is the complement of $C(\Sigma)$. Moreover, $C(\Sigma)$ is a closed subset of $M$ and $C\left(\Sigma^{c}\right)$ is open.

Lemma 4. Let $M$ be an n-dimensional Riemannian manifold with nonnegative Ricci curvature. Then

$$
\lim _{r \rightarrow \infty} r^{-n} \operatorname{Vol}(B(\Sigma, r))=\alpha_{M} \quad \text { and } \quad \operatorname{Vol}(B(\Sigma, r)) \geq \alpha_{M} r^{n} .
$$

Proof. Since $\lim _{r \rightarrow \infty} r^{-n} \operatorname{Vol}(B(p, r))=\alpha_{M}$ and $\operatorname{Vol}(B(p, r))=\operatorname{Vol}(B(\Sigma, r))+$ $\operatorname{Vol}\left(B\left(\Sigma^{c}, r\right)\right)$, it suffices to show that $\lim _{r \rightarrow \infty} r^{-n} \operatorname{Vol}\left(B\left(\Sigma^{c}, r\right)\right)=0$. Given any $\epsilon>0$, choose $\delta>0$ so small such that the open $\delta$-neighborhood $\Sigma_{\delta}$ of $\Sigma$ in $S_{p} M$ satisfies $\operatorname{Vol}\left(\Sigma_{\delta} \backslash \Sigma\right)<\epsilon$. By the Bishop-Gromov volume comparison theorem, $r^{-n} \operatorname{Vol}\left(B\left(\Sigma_{\delta} \backslash \Sigma, r\right)\right)$ is a nonincreasing function of $r$. Thus

$$
\begin{aligned}
r^{-n} \operatorname{Vol}\left(B\left(\Sigma_{\delta} \backslash \Sigma, r\right)\right) & \leq \lim _{r \rightarrow 0} r^{-n} \operatorname{Vol}\left(B\left(\Sigma_{\delta} \backslash \Sigma, r\right)\right) \\
& =n^{-1} \operatorname{Vol}\left(\Sigma_{\delta} \backslash \Sigma\right)<\epsilon / n
\end{aligned}
$$

where $\operatorname{Vol}\left(\Sigma_{\delta} \backslash \Sigma\right)$ is the $(n-1)$-dimensional volume in the unit sphere $S_{p} M$.

The complement $\Sigma_{\delta}^{c}$ of $\Sigma_{\delta}$ is a compact subset in $S_{p} M$ since $\Sigma_{\delta}$ is open and $\Sigma_{\delta}^{c} \cap \Sigma=\emptyset$. Thus there is a constant $r_{0}>0$ such that $B\left(\Sigma_{\delta}^{c}, r\right) \subset B\left(p, r_{0}\right)$ for all $r \geq 0$. Therefore

$$
\begin{aligned}
r^{-n} \operatorname{Vol}\left(B\left(\Sigma^{c}, r\right)\right) & =r^{-n} \operatorname{Vol}\left(B\left(\Sigma_{\delta}^{c}, r\right)\right)+r^{-n} \operatorname{Vol}\left(B\left(\Sigma_{\delta} \backslash \Sigma, r\right)\right) \\
& \leq r^{-n} \operatorname{Vol}\left(B\left(p, r_{0}\right)\right)+\epsilon / n \\
& \leq\left(r_{0}^{n} r^{-n} \operatorname{Vol}\left(S_{p} M\right)+\epsilon\right) / n<\epsilon
\end{aligned}
$$

for $r>r_{0}$ sufficiently large.

Since $\epsilon>0$ is arbitrarily small, we have proved that $\lim _{r \rightarrow \infty} r^{-n} \operatorname{Vol}\left(B\left(\Sigma^{c}, r\right)\right)=$ 0 and therefore $\lim _{r \rightarrow \infty} r^{-n} \operatorname{Vol}(B(\Sigma, r))=\alpha_{M}$. The inequality then follows from this limit and the monotonicity of the function $r^{-n} \operatorname{Vol}(B(\Sigma, r))$. The proof is now completed.

Corollary 5. Let $M$ be as in Lemma 4. Then

$$
\operatorname{Vol}\left(B\left(\Sigma^{c}, r\right)\right)=o\left(r^{n}\right) .
$$

Recall that the excess function $e_{p q}(x)$ with respect to two points $p, q \in M$ is defined by

$$
e_{p q}(x)=d(x, p)+d(x, q)-d(p, q)
$$

where $d(p, q)$ is the distance from $p$ to $q$.

The excess function $e_{p q}$ was introduced by Abresch and Gromoll in 1] and it is instrumental in the proof of finite topological type theorems for complete manifolds with nonnegative Ricci curvature. The key is the following upper bound estimate for $e_{p q}$.

Lemma 6. Let $M$ be a complete $n$-dimensional Riemannian manifold with nonnegative Ricci curvature. Then for any $x \in M$,

$$
e_{p q}(x) \leq 8\left(s^{-1} h^{n}\right)^{1 /(n-1)}
$$

where $s=\min \{d(x, p), d(x, q)\}, h=d(x, \gamma)$, and $\gamma$ is a minimal geodesic from $p$ to $q$. 
The reader is refered to [1] and [2] for a proof.

Lemma 7. Let $M$ be a complete Riemannian manifold with sectional curvature $K \geq-1$. If $x \in M$ is a critical point of the distance function $d(y)=d(y, p)$, $d(x, p) \geq a$, and $d(x, q) \geq a$ with $a=-\frac{1}{2} \ln \left(2^{1 / 4}-1\right)$, then

$$
e_{p q}(x) \geq \ln 2^{1 / 2}>0 .
$$

Proof. The proof is based on the Toponogov comparison theorem [3]. Let $\gamma_{1}$ be a minimal geodesic from $x$ to $q$. Since $x$ is a critical point of the distance function $d(y)=d(y, p)$, there is a minimal geodesic $\gamma_{2}$ from $x$ to $p$ such that $\theta=\angle\left(\gamma_{1}^{\prime}(0), \gamma_{2}^{\prime}(0)\right) \leq \pi / 2$. Let $s_{1}=d(x, q), s_{2}=d(x, p)$, and $t=d(p, q)$. Since $K \geq-1$ and $\theta \leq \pi / 2$, Toponogov's comparison theorem implies that

$$
\cosh t \leq \cosh s_{1} \cosh s_{2}-\cos \theta \sinh s_{1} \sinh s_{2} \leq \cosh s_{1} \cosh s_{2} .
$$

Thus

$$
e^{t} \leq 2 \cosh s_{1} \cosh s_{2}=\frac{1}{2}\left(e^{s_{1}}+e^{-s_{1}}\right)\left(e^{s_{2}}+e^{-s_{2}}\right)
$$

and

$$
\begin{aligned}
e_{p q}(x) & =s_{1}+s_{2}-t \geq \ln 2+s_{1}+s_{2}-\ln \left\{\left(e^{s_{1}}+e^{-s_{1}}\right)\left(e^{s_{2}}+e^{-s_{2}}\right)\right\} \\
& =\ln 2-\ln \left(1+e^{-2 s_{1}}\right)-\ln \left(1+e^{-2 s_{2}}\right) \geq \ln 2-2 \ln \left(1+e^{-2 a}\right) \\
& =\ln 2^{1 / 2}>0
\end{aligned}
$$

\section{An estimate OF THE INSCRIBED RADiUs IN THE COMPLEMENT} OF THE CONE OF RAYS

Given $r>0$, let

$$
h(r)=\max \{d(x, C(\Sigma)) \mid x \in \partial B(p, r)\}
$$

be the maximum distance from points in $\partial B(p, r)$ to the cone of rays from $p$. In the following lemma, we give an estimate of $h(r)$ in terms of the volume growth rate of $B\left(\Sigma^{c}, r\right)$.

Lemma 8. Let $M$ be a complete $n$-dimensional Riemannian manifold with nonnegative Ricci curvature and large volume growth. Assume that $\operatorname{Vol}\left(B\left(\Sigma^{c}, r\right)\right)=o\left(r^{k}\right)$ for some positive constant $k>0$. Then

$$
h(r)=o\left(r^{\frac{k-1}{n-1}}\right) .
$$

In particular, if $\operatorname{Vol}\left(B\left(\Sigma^{c}, r\right)\right)=o\left(r^{2-1 / n}\right)$, then

$$
h(r)=o\left(r^{1 / n}\right) .
$$

Proof. Since $k=2-1 / n$ implies $\frac{k-1}{n-1}=1 / n$, the second part of the lemma follows immediately from the first part.

Suppose that, on the contrary, there is a positive constant $C$ and a divergent increasing sequence of numbers $\left\{r_{l}\right\}_{l=1}^{\infty}$ such that $h_{l} \equiv h\left(r_{l}\right) \geq C r_{l}^{\frac{k-1}{n-1}}$.

Let $x_{l} \in \partial B\left(p, r_{l}\right)$ be a point in $\partial B\left(p, r_{l}\right)$ such that $d\left(x_{l}, C(\Sigma)\right)=h_{l}$ for each $l=1,2,3, \cdots$. Since $M$ is of large volume growth, we have, by Lemma 2 ,

$$
\operatorname{Vol}\left(B\left(x_{l}, h_{l}\right)\right) \geq \alpha_{M} h_{l}^{n}>0 .
$$


By the definition of $h(r)$, we have $B\left(x_{l}, h_{l}\right) \subset C\left(\Sigma^{c}\right)$ and $h_{l} \leq r_{l}$. Thus $B\left(x_{l}, h_{l}\right)$ $\subset B\left(\Sigma^{c}, 2 r_{l}\right)$. It follows from the above inequality and Corollary 5 that $h_{l}=o\left(r_{l}\right)$. The triangle inequality implies that

$$
B\left(x_{l}, h_{l}\right) \subset B\left(\Sigma^{c}, r_{l}+h_{l}\right) \backslash B\left(\Sigma^{c}, r_{l}-h_{l}\right) .
$$

Applying the Bishop-Gromov volume comparison theorem to the pair $B\left(\Sigma^{c}, r_{l}+h_{l}\right)$ and $B\left(\Sigma^{c}, r_{l}-h_{l}\right)$ yields

$$
\frac{\operatorname{Vol}\left(B\left(\Sigma^{c}, r_{l}+h_{l}\right)\right)}{\operatorname{Vol}\left(B\left(\Sigma^{c}, r_{l}-h_{l}\right)\right)} \leq\left[\frac{r_{l}+h_{l}}{r_{l}-h_{l}}\right]^{n}=\left[\frac{1+h_{l} r_{l}^{-1}}{1-h_{l} r_{l}^{-1}}\right]^{n} .
$$

Hence

$$
\begin{aligned}
\alpha_{M} h_{l}^{n} & \leq \operatorname{Vol}\left(B\left(x_{l}, h_{l}\right)\right) \\
& \leq \operatorname{Vol}\left(B\left(\Sigma^{c}, r_{l}+h_{l}\right)\right)-\operatorname{Vol}\left(B\left(\Sigma^{c}, r_{l}-h_{l}\right)\right) \\
& \leq \operatorname{Vol}\left(B\left(\Sigma^{c}, r_{l}-h_{l}\right)\right)\left\{\left[\frac{1+h_{l} r_{l}^{-1}}{1-h_{l} r_{l}^{-1}}\right]^{n}-1\right\} \\
& \leq 2 n r_{l}^{k-1} h_{l} \frac{\operatorname{Vol}\left(B\left(\Sigma^{c}, r_{l}-h_{l}\right)\right)}{r_{l}^{k}}
\end{aligned}
$$

for $l$ sufficiently large.

Thus for large $l$, we have

$$
r_{l}^{-k} \operatorname{Vol}\left(B\left(\Sigma^{c}, r_{l}-h_{l}\right)\right) \geq(2 n)^{-1} \alpha_{M} r_{l}^{1-k} h_{l}^{n-1} \geq(2 n)^{-1} \alpha_{M} C^{n-1}>0 .
$$

This contradicts the volume growth condition $\operatorname{Vol}\left(B\left(\Sigma^{c}, r\right)\right)=o\left(r^{k}\right)$ and the proof is completed.

\section{Proof of Finite topological type}

We shall first prove the following Theorem 9 which implies Theorem 1.

Theorem 9. Let $M$ be a complete n-dimensional Riemannian manifold with nonnegative Ricci curvature and large volume growth. Assume that its conjugate radius is bounded from below by a positive constant or its sectional curvature is bounded from below by a negative constant. If $\operatorname{Vol}\left(B\left(\Sigma^{c}, r\right)\right)=o\left(r^{2-1 / n}\right)$, then $M$ is of finite topological type.

Proof. By rescaling the metric by a positive constant, we can always assume that the sectional curvature satisfies $K \geq-1$ in the case that the sectional curvature is bounded from below by a negative constant.

By the isotopy lemma [2], it suffices to show that the distance function $d(x)=$ $d(x, p)$ has no critical points outside a large ball centered at $p$.

Suppose that, on the contrary, there is a sequence of critical points $\left\{x_{l}\right\}_{l=1}^{\infty} \subset M$ of the distance function $d(x)$ such that $\lim _{l \rightarrow \infty} d\left(x_{l}\right)=\infty$. Notice that $\left\{x_{l}\right\}_{l=1}^{\infty} \subset$ $C\left(\Sigma^{c}\right)$ since no points in the cone of rays $C(\Sigma)$ from $p$ is a critical point of $d(x)$.

Let $r_{l}=d\left(x_{l}\right)$ and $h_{l}=d\left(x_{l}, C(\Sigma)\right), l=1,2,3, \cdots$. Since $\operatorname{Vol}\left(B\left(\Sigma^{c}, r\right)\right)=$ $o\left(r^{2-1 / n}\right)$, it follows from the definition of $h(r)$ and Lemma 8 that $h_{l} \leq h\left(r_{l}\right)=$ $o\left(r_{l}^{1 / n}\right)$.

Since $C(\Sigma)$ is the cone of rays from the base point $p$, there is a ray $\sigma_{l}:[0, \infty) \rightarrow$ $M$ starting from $p$ such that $h_{l}=d\left(x_{l}, \sigma_{l}\right)$. 
Apply the Abresch-Gromoll excess estimate in Lemma 6 to the point $x_{l}$ with $q_{l}=\sigma_{l}\left(2 r_{l}\right)$ to obtain

$$
e_{p q}\left(x_{l}\right) \leq 8\left(r_{l}^{-1} h_{l}^{n}\right)^{1 /(n-1)}=o(1)
$$

since $d\left(q_{l}, x_{l}\right) \geq d\left(p, q_{l}\right)-d\left(p, x_{l}\right)=r_{l}, s_{l}=\min \left\{d\left(x_{l}, p\right), d\left(x_{l}, q_{l}\right)\right\}=r_{l}$, and $h_{l}=d\left(x_{l}, \sigma_{l}\right)=o\left(r_{l}^{1 / n}\right)$. Hence $\lim _{l \rightarrow \infty} e_{p q}\left(x_{l}\right)=0$.

On the other hand, if the sectional curvature $K \geq-1$, then it follows from Lemma 7 that

$$
e_{p q}\left(x_{l}\right) \geq \ln 2^{1 / 2}>0
$$

for all $l$ sufficiently large; if the conjugate radius is bounded from below by a positive constant, then the same argument as in [6] shows that $e_{p q}\left(x_{l}\right)$ is uniformly bounded from below by a positive constant. In either case, we have a contradiction. Therefore, there is no critical point of the distance function $d(x)=d(x, p)$ outside a large ball $B(p, r)$ and $M$ is of finite topological type.

Proof of Theorem 1. Lemma 2 implies that $\alpha=\alpha_{M}$. Since $B(p, r)$ is the disjoint union of $B(\Sigma, r)$ with $B\left(\Sigma^{c}, r\right)$, we have $V(B(p, r))=\operatorname{Vol}(B(\Sigma, r))+\operatorname{Vol}\left(B\left(\Sigma^{c}, r\right)\right)$. It follows from Lemma 4 that

$$
\begin{aligned}
\operatorname{Vol}\left(B\left(\Sigma^{c}, r\right)\right) & =\operatorname{Vol}(B(p, r))-\operatorname{Vol}(B(\Sigma, r)) \\
& =\alpha_{M} r^{n}+o\left(r^{2-1 / n}\right)-\operatorname{Vol}(B(\Sigma, r)) \leq o\left(r^{2-1 / n}\right) .
\end{aligned}
$$

Thus Theorem 1 follows from Theorem 9.

\section{REFERENCES}

[1] U. Abresch and D. Gromoll, On complete manifolds with nonnegative Ricci curvature, J. AMS 3 (1990) 41-55. MR 91a:53071

[2] J. Cheeger, Critical points of distance functions and applications to geometry, Lecture Notes in Math., Springer-Verlag 1504 (1991) 1-38. MR 94a:53075

[3] J. Cheeger and D. Ebin, Comparison theorems in Riemannian geometry, North-Holland, Amsterdam (1975). MR 56:16538

[4] J. Cheeger and D. Gromoll, On the structure of complete manifolds of nonnegative curvature, Annals of Math. 96, 413-443 (1972). MR 46:8121

[5] X. Menguy, Noncollapsing examples with positive Ricci curvature and infinite topological type, preprint.

[6] Z. M. Shen, Complete manifolds with nonnegative Ricci curvature and large volume growth, Inventiones mathematicae (1996) 393-404. MR 97d:53045

[7] Z. M. Shen and G.F. Wei, Volume growth and finite topological type, Differential geometry: Riemannian geometry (Los Angeles, CA, 1990), 539-549, Proc. Sympos. Pure Math., 54, Part 3, Amer. Math. Soc., Providence, RI, 1993. MR 94e:53036

[8] J. P. Sha and D. G. Yang, Examples of manifolds of positive Ricci curvature, J. Differ. Geom. 29 (1989) 95-103. MR 90c:53110

[9] J. P. Sha and D. G. Yang, Positive Ricci curvature on the connected sum of $S^{n} \times S^{m}$, J. Differ. Geom. 33 (1991) 127-137. MR 92f:53048

Department of Mathematics, Harvard University, Cambridge, Massachusetts 02139

E-mail address: ordway@abel.math.harvard.edu

E-mail address: bstephen@fas.harvard.edu

Department of Mathematics, Tulane University, New Orleans, Louisiana 70118

E-mail address: dgy@math.tulane.edu 